\title{
DEFECTS IN DECOMPOSING COPPER ALLOYS STUDIED BY POSITRONS
}

\author{
V.S. Mikhalenkov \\ Institute of Metal Physics, Ukrainian Academy of Sciences, 252680 Kiev-142, Ukraine \\ A.P. De Lima and C. Lopes GiL \\ Physics Department, University of Coimbra, 3000 Coimbra, Portugal
}

\begin{abstract}
The evolution of defect structure during isochronal annealing of water-quenched $\mathrm{Cu}-4.83$ at.\% In and $\mathrm{Cu}-4.94$ at.\% Sn alloys was studied by measuring positron lifetime and Doppler broadening of annihilation line. The behaviour of annihilation parameters in both alloys is found to be analogous. In as-quenched alloys one component of about $180 \mathrm{ps}$ is detected in the lifetime spectra. It remains intact up to the annealing temperature of $450 \mathrm{~K}$ and is attributed to positron annihilation at quenched-in vacancies associated with alloying atoms. When going above this temperature, the short component appears in the lifetime spectra, while intensity of the long component starts to drop giving evidence to the reduction of defect concentration. This process is observed to develop up to $550 \mathrm{~K}$; thereafter all annihilation parameters level off. After crossing the phase separation temperature they begin to vary again since the defect structure comes into dependence on cooling conditions. The obtained results are discussed in terms of defect structure evolution in the course of precipitate formation.
\end{abstract}

PACS numbers: 78.70.Bj, 81.30.Mh

\section{Introduction}

Decomposition of supersaturated solid solutions (hereafter referred to as DSSS) forms the basis for a range of heat treatment methods intended for improving the mechanical properties of metallic materials due to generation of dispersed, both coherent and incoherent, precipitates. In particular, aging of quenched alloys is among these methods. Lattice defects are directly involved in DSSS process providing both alloying atoms transport to the growing precipitates and accommodation of precipitates in the parent lattice. On the other hand, defects can be produced in the DSSS process itself, e.g. in the course of discontinuous precipitation and through the formation of interphase boundaries.

A knowledge of defect evolution in the act of DSSS would gain a better insight into DSSS mechanisms at different stages of process. Cu-based alloys make 
convenient choice for such investigations as DSSS in these alloys proceeds at moderate temperatures such as to retain a good deal of non-equilibrium defects during the processes involved. Positron spectroscopy has been established to be a suitable method for studying atomic-size defects in metals. It is especially sensitive to the concentration and atomic environment of vacancies, their agglomerates and complexes with impurities and alloying atoms [1].

Variations of positron spectra during annealing of decomposing $\mathrm{Cu}-\mathrm{Sn}$ and $\mathrm{Cu}-\mathrm{In}$ alloys have been ever studied in the papers $[2,3]$. In quenched $\mathrm{Cu}-4.9$ at.\% $\mathrm{Sn}$ alloy positron lifetime (PLT) spectra measured in the course of isochronal annealing were fitted in terms of single-component analysis [2]. In Ref. [3] the lineshape parameter of Doppler broadened annihilation line was measured in slowly and rapidly cooled down $\mathrm{Cu}-3.3$ at.\% In alloy as a function of annealing temperature, and a marked difference between these two kinds of cooling conditions has been discovered. In this paper we utilized both PLT and Doppler broadening of annihilation line (DBAL) techniques with the goal of comparative tracing the evolution of defect structure in quenched $\mathrm{Cu}-4.83$ at.\% In and $\mathrm{Cu}-4.94$ at.\% Sn alloys in the course of isochronal annealing performed under identical conditions.

\section{Experimental}

The alloys were prepared from $5 \mathrm{~N}$ components by arc-melting in purified Ar atmosphere. The compositions were verified by X-ray spectroscopy. The sample preparation included homogenization of the ingots at $1125 \mathrm{~K}$ for $24 \mathrm{~h}$ and cutting. After keeping for $0.5 \mathrm{~h}$ at $925 \mathrm{~K}$ in Ar atmosphere the samples were quenched in water. Isochronal $(0.5 \mathrm{~h})$ annealing was performed in vacuum of $0.26 \mathrm{mPa}$ at sequentially increasing temperatures up to $825 \mathrm{~K}$. Each treatment was followed by PLT and DBAL measurements. Positron source of $2 \mathrm{MBq}{ }^{22} \mathrm{NaCl}$ sealed into Kapton foil of $1 \mathrm{mg} / \mathrm{cm}^{2}$ thick was sandwiched between two identical samples.

PLT spectra were taken at room temperature using conventional fast-fast coincidence system with a time resolution of $240 \mathrm{ps}$ (FWHM). More than $2 \times 10^{6}$ events were accumulated in each spectrum. After background and source corrections, the spectra were analyzed by taking use of POSITRONFIT programme [4]. The average lifetime, $\tau_{\mathrm{av}}$, was calculated as $\tau_{\mathrm{av}}=I_{1} \tau_{1}+I_{2} \tau_{2}$, where $\tau_{1,2}$ are PLT's of short and long components and $I_{1,2}$ are their intensities. DBAL spectra were measured by means of intrinsic Ge detector with energy resolution of $1.24 \mathrm{keV}$ at ${ }^{103} \mathrm{Ru}$ peak. The position of annihilation peak was stabilized by monitoring the system with ${ }^{103} \mathrm{Ru} \gamma$-line. DBAL lineshape was analyzed in terms of $S$ - and $W$-parameters determined as the normalized areas in a fixed number of channels in the central part and at the wings of spectrum, respectively. The n!lmber of fixed channels was chosen so as to get for as-quenched samples $S$ value close to 0.5 and $W$ value close to 0.2 .

\section{Results}

Variations of average lifetimes, $\tau_{\mathrm{av}}$, and of $S$-parameters with the annealing temperature, $T_{\text {an }}$, for the studied $\mathrm{Cu}-\mathrm{Sn}$ and $\mathrm{Cu}-\mathrm{In}$ alloys are plotted in Fig. 1a and $\mathrm{b}$, respectively. Apart from some details, the annealing behaviour of $\tau_{\mathrm{av}}$ and 

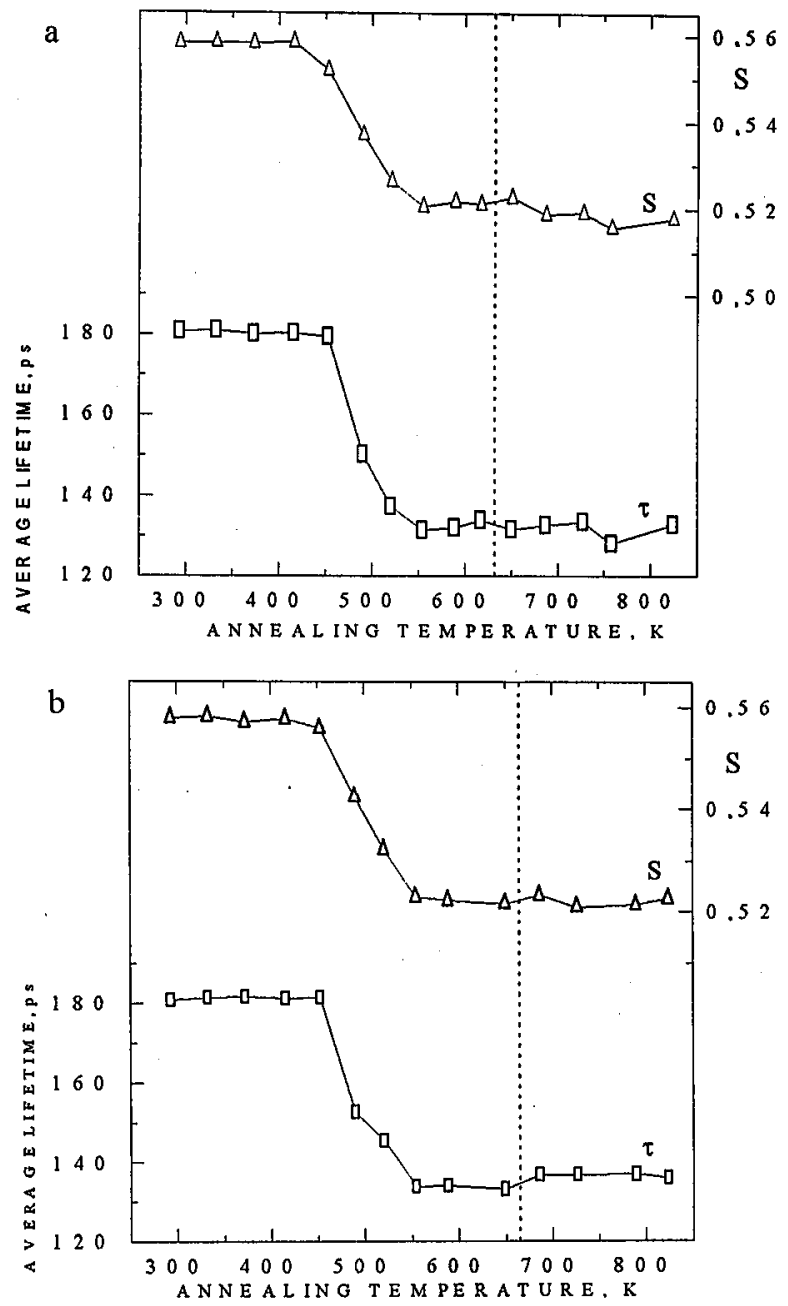

Fig. 1. Average positron lifetime (squares) and $S$-parameter (triangles) in $\mathrm{Cu}-4.94$ at.\% Sn (a) and 4.83 at.\% In (b) alloys vs. annealing temperature. The phase separation temperatures are marked by vertical dotted lines. The lines are guides to an eye.

$S$ is similar in both alloys. Up to $T_{\mathrm{an}}=450 \mathrm{~K}$ both characteristics remain almost unchanged. Their main changes occur in the range of $T_{\mathrm{an}}$ from $450 \mathrm{~K}$ to $550 \mathrm{~K}$, where $\tau_{\mathrm{av}}$ drops to the levels of $135 \mathrm{ps}$ in $\mathrm{Cu}-\mathrm{In}$ and of $133 \mathrm{ps}$ in $\mathrm{Cu}-\mathrm{Sn}$ alloy. These values are higher than PLT in well-annealed Cu (about 120 ps). Above $T_{\mathrm{an}}=550 \mathrm{~K} \tau_{\mathrm{av}}$ levels off and remains nearly intact up to borderline between two-phase and one-phase regions which are located, according to the phase diagrams [5], at $635 \mathrm{~K}$ and $715 \mathrm{~K}$ for $\mathrm{Cu}-\mathrm{Sn}$ and $\mathrm{Cu}-\mathrm{In}$ alloys, respectively. Above these temperatures the structure and, hence, the annihilation characteristics get dependent on the overheating the alloys in single-phase region and on the cooling conditions in two-phase one. 

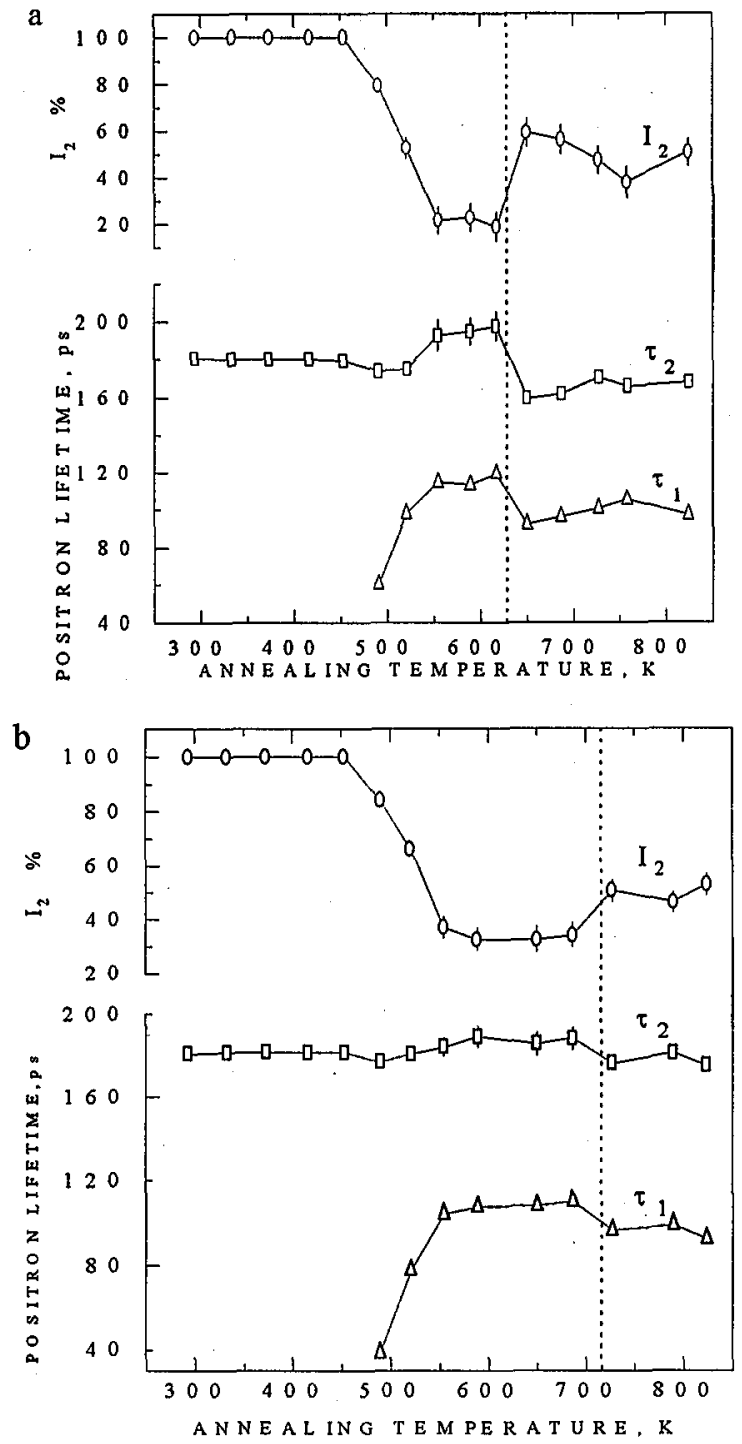

Fig. 2. Positron lifetime components $\tau_{1}$ (triangles), $\tau_{2}$ (squares) and the intensity of long component $I_{2}$ (circles) in $\mathrm{Cu}-4.94$ at.\% $\mathrm{Sn}$ (a) and $\mathrm{Cu}-4.83$ at.\% In (b) alloys vs. annealing temperature. The phase separation temperatures are marked by vertical dotted lines. The lines are guides to an eye.

Figure $2 \mathrm{a}$ and $\mathrm{b}$ displays the variations of the short lifetime, $\tau_{1}$, long lifetime, $\tau_{2}$, and its intensity, $I_{2}$, in $\mathrm{Cu}-\mathrm{Sn}$ (a) and $\mathrm{Cu}-\mathrm{In}$ (b) alloys as the functions of annealing temperature. Below $T_{\text {an }}=450 \mathrm{~K}$ only $\tau_{2}$ component of about $180 \mathrm{ps}$ is observed in the spectra for both alloys. This value is close to that observed in electron-irradiated $\mathrm{Cu}$ [6] where all positrons were proved to annihilate being trapped at vacancies. Above $T_{\mathrm{an}}=450 \mathrm{~K}$ the short component $\tau_{1}$ comes into 
view. Its magnitude and intensity are increasing with $T_{\text {an }}$ approaching the values of $\tau_{1}=120 \mathrm{ps}$ and $I_{1}=80 \%$ in Cu-Sn alloy and $\tau_{1}=110 \mathrm{ps}$ and $I_{1}=70 \%$ in $\mathrm{Cu}-\mathrm{In}$ one. Above $T_{\text {an }}=550 \mathrm{~K}$ lifetimes and their intensities are nearly constant up to the phase separation temperatures. Crossing these temperatures causes reduction in $\tau_{1}$ and $\tau_{2}$ and the increase in $I_{2}$. The PLT components, that might have been attributed to vacancy clusters, have not been detected.

\section{Discussion}

The analogy in annealing behaviour of annihilation characteristics in $\mathrm{Cu}-\mathrm{In}$ and $\mathrm{Cu}-\mathrm{Sn}$ alloys testifies to the similarity of defect evolution in the course of their decomposition. The only type of positron traps in the as-quenched alloys must be non-equilibrium vacancies. As the grain size amounted to several tenths of $\mathrm{mm}$, we disregarded the contribution of positron trapping at grain boundaries. The main peculiarity of the early annealing stage is one-component PLT spectra which is retained up to $450 \mathrm{~K}$. As vacancies in pure $\mathrm{Cu}$ get mobile and start to anneal out as early as $250 \mathrm{~K}$ [7], their survival well above the room temperature has to be assigned to stabilizing action of alloying atoms through the formation of complexes with vacancies. A considerable difference in atomic radii between $\mathrm{Cu}$ and In or Sn, which exceeds $30 \%$ [8], promotes vacancy to incorporate at least one alloying atom into its environment. The values of vacancy binding energy with In and $\mathrm{Sn}$ atoms have not been found in the literature but they should be of the same order of magnitude as in $\mathrm{Cu}-\mathrm{Ga}$ and $\mathrm{Cu}-\mathrm{Ge}$ alloys, where vacancy binding energies with $\mathrm{Ga}$ and $\mathrm{Ge}$ atoms amount to $0.18 \mathrm{eV}$ and $0.28 \mathrm{eV}$, respectively [9]. Therefore we can conclude that at the early annealing stage the vacancy-alloying atoms complexes serve as the positron traps.

The appearance of short PLT component (see Fig. 2), as well as reduction of $\tau_{\mathrm{av}}$ and $S$ (Fig. 1), at annealing temperatures above $450 \mathrm{~K}$ indicate that a fraction of positrons, $\eta$, begins to annihilate from the free state. This testifies to the decrease in defect concentration, $C_{\nu}$. According to the trapping model [1], $\eta$, is defined by the relation: $\eta=\lambda_{\mathrm{f}} /\left(\lambda_{\mathrm{f}}+k\right)$, where $\lambda_{\mathrm{f}}$ is the annihilation rate for free positron and the trapping rate $k$ is proportional to $C_{\nu}: k=\mu C_{\nu}$, where $\mu$ stands for specific trapping rate. The short lifetime component is related to $k$ as $\tau_{1}=\tau_{\mathrm{f}} /\left(1+k \tau_{\mathrm{f}}\right)$, where $\tau_{\mathrm{f}}=\lambda_{\mathrm{f}}^{-1}$ is PLT in defect-free crystal. It is evident that the increase in $\tau_{1}$ is conditioned by the reduction of $k$. Thus PLT data enables defect concentration be calculated from the equation

$$
C_{\nu}=\frac{k}{\mu}=\frac{I_{2}\left(\tau_{2}-\tau_{1}\right)}{\mu \tau_{1} \tau_{2}}
$$

if $\mu$ for the particular type of defect is known.

It is seen from Fig. $2 \mathrm{a}$ and $\mathrm{b}$ that the annealing above $450 \mathrm{~K}$ causes reduction of $I_{2}$ in both alloys. As the precipitates are formed just in this temperature range, we attribute such behaviour of $I_{2}$ to migration of vacancy-alloying atom complexes to the growing precipitates. Some increase in $\tau_{2}$ may be assigned to dissociation of these complexes.

To give an insight into precipitate growth mechanism incorporating vacancyalloying atom complexes "a vacancy pump model" has been suggested [10]. In this 
model the complexes are supposed to migrate to precipitate nuclei and dissociate at the interphase boundary. After giving up the alloying atom to the nucleus, vacancy turns up to the bulk where it picks up another alloying atom, and the process is repeated. In this case vacancy concentration appears to be kept unchanged. However, the model is based on an assumption that the specific volumes of the bulk and precipitates are not too distinguished. In the alloys considered here the specific volume of growing phases exceeds considerably that of the bulk. This produces at the interphase boundaries significant elastic stresses which may be reduced with the aid of vacancies. Therefore the "vacancy pump model" does not work exactly in our case. More likely the vacancy, after having delivered the alloying atom to a nucleus, is left at the interphase boundary promoting relaxation of interphase stresses. This situation corresponds to some reduction in vacancy concentration, as the vacancies are spent for accommodation of precipitates.

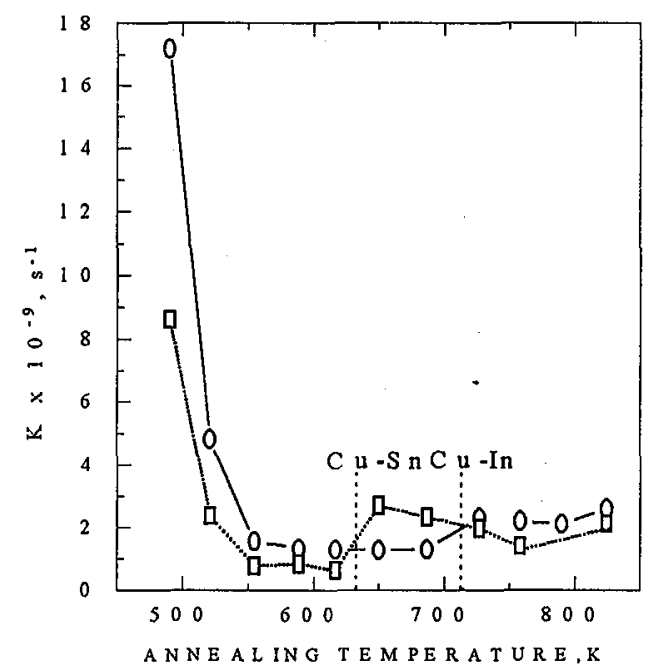

Fig. 3. Positron trapping rate in $\mathrm{Cu}-4.94$ at.\% $\mathrm{Sn}$ (squares) and $\mathrm{Cu}-4.83$ at.\% In (circles) alloys as a function of annealing temperature. The phase separation temperatures are marked by dotted lines. The lines are guides to an eye.

Figure 3 displays dependence of the trapping rate on annealing temperature: Below $550 \mathrm{~K} k$ decreases in both alloys reflecting a reduction in defect concentration. Above $550 \mathrm{~K} \tau_{1}, \tau_{2}$ and $I_{2}$ level off (see Fig. 2). In doing so $I_{2}$ holds the value close to $20 \%$ and $30 \%$ in $\mathrm{Cu}-\mathrm{Sn}$ and $\mathrm{Cu}-\mathrm{In}$, respectively, up to the temperature of transition to the $\alpha$-phase region. As the magnitude of $k$ remains nearly constant from $550 \mathrm{~K}$ to this temperature, one may conclude that defect concentration is unchanged in this range. The values of $\mu$ for $\mathrm{Cu}-\mathrm{In}$ and $\mathrm{Cu}-\mathrm{Sn}$ alloys are not available. For pure $\mathrm{Cu} \mu$ is known to amount to $4.3 \times 10^{14} \mathrm{~s}^{-1}$ [11]. Assuming that alloying does not affect $\mu$ noticeably, we have taken use of $\mu$ in pure $\mathrm{Cu}$ for rough evaluation of defect concentration in the alloys under study. Then $C_{\nu}$ appears to drop during annealing in the range from $450 \mathrm{~K}$ to $550 \mathrm{~K}$ from $4 \times 10^{-5}$ to $0.3 \times 10^{-5}$ 
and from $2 \times 10^{-5}$ to $0.14 \times 10^{-5}$ in $\mathrm{Cu}-\mathrm{In}$ and $\mathrm{Cu}-\mathrm{Sn}$ alloys, respectively. Leveling off the defect concentration above $550 \mathrm{~K}$ points to stabilization of defect structure.

At elevated annealing temperature decomposition mechanism may be changed from continuous to discontinuous which is characterized by precipitate nucleation at the grain boundaries and by vacancies generation during the precipitates growth. In $\mathrm{Cu}-4.8$ at.\% In alloy such change of the mechanism was found to occur at $620 \mathrm{~K}$ [12]. There is indication on the possibility of discontinuous mechanism also in $\mathrm{Cu}-\mathrm{Sn}$ system [13]. It is seen in Fig. 2b that in $\mathrm{Cu}-\mathrm{In}$ alloy $I_{2}$ remains unaltered near $620 \mathrm{~K}$. This means that transition to another mechanism does not create detectable amount of additional positron traps since the vacancies formed in the act of discontinuous decomposition are consumed at growing precipitates. The same is also true for the loss of coherency by precipitates. As both $\tau_{2}$ and $I_{2}$ are nearly constant from $550 \mathrm{~K}$ up to the boundary of $\alpha$-phase region, the contribution of incoherent interfaces may be thought to be insignificant in forming extra positron traps.

To identify defects which trap positrons, $R$-parameter [14] is commonly employed. It is defined from Doppler broadened annihilation lines for defected and perfect materials and is insensitive to the defect concentration, depending on the type of defects only. We determined $R$-parameter by taking advantage of the method suggested in [3], where this parameter is calculated from the slope of relation between $S$ - and $W$-parameters: $R=S\left(T_{\text {an }}\right) / W\left(T_{\text {an }}\right)$. Calculated in this way $R$-parameter does not depend on the temperature and amounts to 0.970 and to 0.935 in $\mathrm{Cu}-\mathrm{In}$ and $\mathrm{Cu}-\mathrm{Sn}$ alloys, respectively. Constancy of $R$-parameter testifies to the fact that only one type of defects dominates in the whole range of annealing temperatures. Besides of vacancy-alloying atom complexes, relaxed vacancies at the growing precipitate boundaries and free vacancies emitted during discontinuous decomposition may contribute to positron trapping. These defects do not differ much in size, and $R$-parameter remains unaltered.

Above $T_{\mathrm{an}}=550 \mathrm{~K} \tau_{2}$ in both alloys approaches $190 \mathrm{ps}$ that correlates with $\tau$ value in electron irradiated $\mathrm{Cu}$. This fact enables us to conclude that positrons are trapped mainly at free vacancies in this range of annealing temperatures. At the same time the highest levels of $\tau_{2}$ are somewhat different in $\mathrm{Cu}-\mathrm{Sn}$ and $\mathrm{Cu}-\mathrm{In}$ alloys (by $8 \mathrm{ps}$ ). This suggests that the atomic surroundings of positron traps in these two alloys are different. Therefore the traps should be thought to reside outside of the region depleted in alloying atoms. However, the existence of extra vacancies inside precipitate is scarcely probable as they should cause additional stresses. More likely, the positron traps in the temperature range considered are vacancies localized near the boundaries of incoherent precipitates or inside these boundaries.

Crossing the point between two- and one-phase regions leads to decrease in $\tau_{1}$ and $\tau_{2}$ and to increase in $I_{2}$ (Fig. 2). This effect must depend upon the extent to which precipitates are dissolved during the stay of alloys in one-phase region. The further decomposition proceeds under condition of gradually decreasing diffusivity during cooling the alloys down. Therefore, the produced defect structure has to be unlike that formed in the course of stepped annealing. As follows from Fig. 3 crossing the phase separation temperature results in twofold and fourfold increase 
in trapping rate in $\mathrm{Cu}$-In and $\mathrm{Cu}-\mathrm{Sn}$ alloys, respectively. As the $R$-parameters do not vary therewith markedly, the type of defects can be thought to remain the same. This means that specific trapping rate, $\mu$, is kept unaltered, and the rise of trapping rate is caused by increase in defect concentration. The reduction in $\tau_{2}$ may be assigned to the fact that ceaseless cooling down from one-phase region is more favourable to the formation of vacancy-alloying atom complexes than the stepped annealing. Therefore, the defect structures formed in the course of stepped annealing of quenched alloy and during continuous cooling down from one-phase region are different. In the last case a comprehensive consideration of defect production requires for cooling conditions to be verified thoroughly. This requirement has not been met in this work. Therefore the evolution of defect structure in the alloys overheated above phase separation temperature is not discussed here.

\section{References}

[1] Positron Solid State Physics, Ed. W. Brandt, A. Dupasquier, North-Holland, Amsterdam 1983.

[2] V.S. Mikhalenkov, P. Hautojärvi, F. Plazaola, Mater. Sci. Forum 105-110, 1153 (1992).

[3] W. Lühr-Tank, A. Sager, H. Bosse, J. Phys. F 17, 827 (1987).

[4] P. Kirkegaard, M. Eldrup, O.E. Mogensen, N.J. Pedersen, Comput. Phys. Commun. 23, 307 (1981).

[5] Binary Alloys Phase Diagrams, Ed. B. Massalski, Vol. 1, Amer. Soc. for Metals, Metals Park 1986.

[6] H.-E. Shaefer, Phys. Status Solidi A 102, 47 (1987).

[7] B. Lengeler, S. Mantl, W. Triftshäuser, J. Phys. F 8, 1691 (1978).

[8] E.B. Royce, Phys. Rev. 164, 929 (1967).

[9] P.N. Diderichs, T. Hoshino, B. Drittler, K. Abraham, R. Zeller, Physica B 172, 203 (1991).

[10] L.A. Girifalko, H. Herman, Acta Metall. 13, 583 (1965).

[11] S. Mantl, W. Kesternich, W. Triftshäuser, J. Nucl. Mater. 69-70, 593 (1975).

[12] L.M. Shapiro, J.S. Kirkaldy, Acta Metall. 16, 1239 (1968).

[13] H. Böhm, Zs. Metallk. 52, 564 (1961).

[14] W. Triftshäuser, Phys. Rev. B 12, 4634 (1975). 